\title{
Pulse Sequences for High-Energy Terahertz Generation
}

\author{
K. Ravi ${ }^{1,4}$, F. Ahr ${ }^{1,2}$, G. Cirmi ${ }^{1,2}$, M. Hemmer ${ }^{1}$, F. Reichert ${ }^{2}$, D. N.Schimpf ${ }^{1,2}$, H. Çankaya ${ }^{1,2}$, O. D. \\ Mücke $^{1,2}$, L. E. Zapata ${ }^{1}$, N. H. Matlis ${ }^{1}$, and F. X. Kärtner ${ }^{1,2,3}$ \\ ${ }^{1}$ Center for Free-Electron Laser Science, DESY, Hamburg, Germany \\ ${ }^{2}$ The Hamburg Center for Ultrafast Imaging and Department of Physics, Hamburg, Germany \\ ${ }^{3}$ Department of EECS and RLE, Massachusetts Institute of Technology, Cambridge, USA
}

\begin{abstract}
A family of approaches employing sequences of optical pump pulses, yielding energy conversion efficiencies in the $5-10 \%$ range, is introduced. A method to generate these sequences by cascaded optical parametric amplification of narrowband pulses is discussed.
\end{abstract}

\section{INTRODUCTION}

$\mathrm{M}$ ulti-cycle terahertz $(\mathrm{THz})$ pulses with frequencies between 0.1 and $1 \mathrm{THz}$ exhibiting simultaneously high energies $(>10 \mathrm{~mJ})$ and peak fields $(>100 \mathrm{MV} / \mathrm{m})$ are exciting prospects for transforming nonlinear $\mathrm{THz}$ spectroscopy, radar and charged particle acceleration [1] applications. Such a class of sources has the potential to ubiquitize charged-particle accelerator technology and enable unprecedented modalities in biomedical imaging, therapy and protein structure analysis [2].

Due to the increase in pulse energies produced by solidstate lasers, laser-driven $\mathrm{THz}$ generation methods based on difference-frequency generation (DFG) or optical rectification (OR) have become attractive. However, an impending problem is the realization of high optical-to- $\mathrm{THz}$ energy conversion efficiencies. For realistic Joule-class lasers, conversion efficiencies of $>>1 \%$ are necessary for the required $\mathrm{THz}$ sources. On the contrary, conversion efficiencies for narrowband $\mathrm{THz}$ sorces have been limited to $0.1 \%$ [3]. In principle, repeated down-conversion of the laser pump frequency or cascaded DFG [4] can result in conversion efficiencies as large as $10 \%$. However, despite being proposed conceptually, there have been neither experimental demonstrations nor feasible designs of such schemes.

Here, we introduce a family of approaches based on cascaded DFG of a sequence of optical pump pulses separated in time by the inverse of the $\mathrm{THz}$ frequency in periodically poled lithium niobate (PPLN) at cryogenic temperatures[5]. The approach is able to circumvent walk-off between optical pump and generated $\mathrm{THz}$ radiation, alleviate laser-induced damage and produce conversion efficiencies between 5-10\%. Methods to generate these sequences by direct generation of pulse trains or a phase-locked set of frequency lines are discussed.

Furthermore, a method to self-generate these pulse sequences [6], by inputing a strong narrowband (100 ps-1 ns transform limited duration) optical pump pulse and frequencyshifted seed pulse (with $0.1 \%$ of pump energy) is described along with initial experiments [7]. The initially generated $\mathrm{THz}$ by the DFG between pump and seed then drives cascaded $\mathrm{THz}$ generation and produces a comb of optical lines in frequency space or a sequence of optical pulses in time. The resulting $\mathrm{THz}$ energy growth is exponential and represents a new approach known as cascaded optical parametric amplification (COPA). COPA is characterized by physics distinctly different from conventional OPAs due to the coupled interaction of multiple triplets of pump, signal and idler waves and is predicted to yield conversion efficiencies of $8 \%$.

\section{Methods And Results}

Numerical simulations account for cascading effects, selfphase modulation, $\mathrm{THz}$ absorption and material dispersion. The refractive indices of lithium niobate in the optical region are obtained from [8] while the $\mathrm{THz}$ refractive indices and absorption coefficients are obtained from [9]-[10]. The second order nonlinearity of lithium niobate is assumed to be $\chi^{(2)}$ $=336 \mathrm{pm} / \mathrm{V}$ and the nonlinear refractive index is set to be $1.25 \times 10^{-19} \mathrm{~m}^{2} /$ Watt. The periodic inversion of $\chi^{(2)}$ is considered explicitly, consequently accounting for all quasiphase-matched $\mathrm{THz}$ wave generation processes. The conversion efficiency for a train of $N$ pulses of full-width at half-maximum (FWHM) duration $\tau$ each separated by the inverse of the $\mathrm{THz}$ frequency, shows values exceeding $6 \%$ at cryogenic temperatures (Fig.1a). Corresponding focused fields (Fig. 1b) approach $\mathrm{GV} / \mathrm{m}$ values. The optimal number of pulses in Fig.1a corresponds to a value $L\left(n_{T H z}-n_{g}\right) / c / T_{0}$, where $T_{0}=f_{T H z}^{-1}$ or the time period of the phase-matched $\mathrm{THz}$ wave. This condition corresponds to a situation when the overlap between the sequence of optical pump pulses is maximized while the overall absorption experienced by the terahertz pulse is minimized. The conversion efficiency and peak electric fields attainable are larger for larger $\mathrm{THz}$ frequencies. However, since absorption for larger $\mathrm{THz}$ frequencies is higher, the conversion efficiency and maximum peak fields will eventually saturate at large $\mathrm{THz}$ frequencies $>$ $1 \mathrm{THz}$.
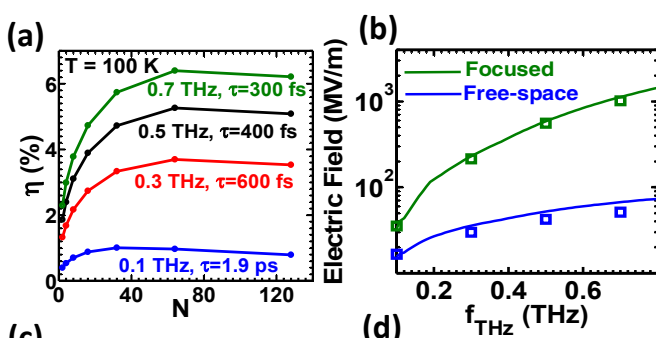

(c)
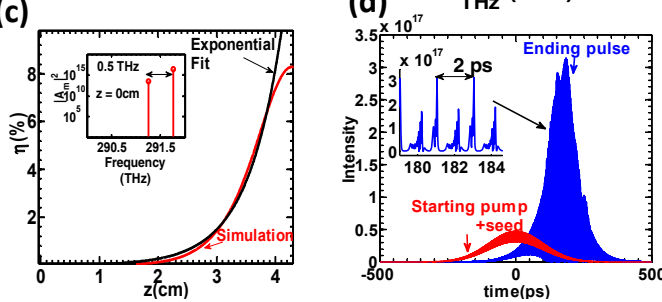

Fig.1(a) Conversion efficiency $\eta$ as a function of the number of pulses $\mathrm{N}$ in a train of pulses with duration $\tau$ for various $\mathrm{THz}$ frequencies; (b) corresponding electric fields; (c) COPA yielding $\eta=8 \%$ at $T=100 \mathrm{~K}$, starting with a strong pump and red-shifted seed with $0.1 \%$ of pump energy (inset); (d) self-generation of a sequence of pulses by COPA. 
While a sequence of pulses offers solutions to obtaining very large terahertz pulse energies and conversion efficiencies, the matter of generating such a sequence of pulses is non-trivial. Several approaches to generating such a sequence of pulses exist. For instance, a single ultrafast pulse can be split and delayed several times to obtain a sequence of pulses. Alternatively, a series of narrowband lines with transform limited durations of several $100 \mathrm{ps}$ in the frequency domain is also tantamount to a sequence of pulses in the time domain. However, obtaining a series of lines at high pump energy is challenging due to limitations of the gain bandwidth of high energy amplifiers.

However, we identified that by starting with a seed pulse, red or blue-shifted with respect to the pump pulse by the requisite terahertz frequency and containing only a fraction of the pump energy, i.e. as low as only $0.1 \%$ can automatically self-generate a series of frequency lines due to the cascading process. In this case, the initial DFG between the pump and the seed results in the generation of terahertz radiation which then drives the generation of further red-shifted spectral lines. The process quickly results in the generation of several spectral lines, leading to exponential growth of terahertz energy as shown in Fig.1c for the case of $0.5 \mathrm{THz}$ generation. Since this approach requires narrowband high energy lasers, it is highly accessible with existing laser technology. Contrary to conventional three wave mixing optical parametric amplifiers, the seed can be either blue or red-shifted with respect to the pump. This is because the initial spectrum very quickly washes out due to the cascading process and therefore the input spectrum is essentially inconsequential.

In the time domain, the initial optical pulse (pump+seed) with weak modulations (Fig. 1d, red) is then converted to a pulse sequence with a periodicity corresponding to the $\mathrm{THz}$ frequency (Fig. 1d+inset, blue). This is because the increased bandwidth of the spectrum caused by cascading leads to the strengthening of the modulations to an extent which eventually leads to the creation of several isolated pulses.

\section{CONCLUSION AND FUTURE WORK}

A family of approaches based on a sequence of pulses was introduced. A method to self-generate these pulse sequences by cascaded optical parametric amplification starting with easily accessible narrowband lasers was described. Conversion efficiencies of 5-10\% and peak fields approaching the Giga Volt $/ \mathrm{m}$ are predicted for $\mathrm{THz}$ pulses with durations of hundreds of picoseconds. This class of approaches promises to generate terahertz pulse energies on the order of $10-100 \mathrm{~mJ}$ in the near future with applications in charged particle acceleration and coherent X-ray generation.

\section{REFERENCES}

[1]E. A. Nanni et al., "Terahertz-driven linear electron acceleration," Nat. Comm. 6:8486 (2015).

[2]F.X.Kärtner, "AXSIS: Exploring the frontiers in attosecond X-ray Science," Nuclear Instruments and Methods in Physics Research A (2016) http://dx.doi.org/10.1016/j.nima.2016.02.080

[3]S.Carbajo et al., "Efficient narrowband terahertz generation in cryogenically cooled periodically poled lithium niobate," Opt. Lett. 40, 5762 (2015).

[4]M. C. Golomb, "Cascaded nonlinear difference frequency generation of enhanced wave production," Opt. Lett. 29, 2046 (2004).
[5] K.Ravi et al, "Pulse Sequences for Efficient Multi-Cycle Terahertz Generation in Periodically Poled Lithium Niobate", arXiv 1606.05278. [6] K.Ravi et al "Cascaded parametric amplification for Highly Efficient Terahertz Generation", arXiv: 1604.08919

[7] M. Hemmer et al., "THz-wave generation via cascaded optical parametric amplification", IRMMW 2016 (Accepted).

[8] D. H. Jundt, "Temperature-dependent Sellmeier equation for the index of refraction, ne, in congruent lithium niobate," Opt. Lett. 22(20), 1553-1555 (1997).

[9] L. Palfalvi et al, "Temperature dependence of the absorption and refraction of Mg-doped congruent and stoichiometric lithium niobate in the THz range," J. App. Phys. 97, 123505 (2005).

[10] J. A. Fulop, et al, "Towards generation of mJ-level ultrashort $\mathrm{THz}$ pulses by optical rectification," Opt. Express 19(16), 15090-15097 (2011). 